\title{
Construcción identitaria y expectativas de estudiantes universitarios indígenas
}

\section{Identity Construction and Expectations of Indigenous University Students}

Rosa Guadalupe Mendoza Zuany (*) lupitamendoza.zuany@gmail.com

(*) Universidad Veracruzana

(Recibido: 8 de mayo de 2015; aceptado para publicación: 1 de agosto de 2016)

Cómo citar: Mendoza, R. G. (2018). Construcción identitaria y expectativas de estudiantes universitarios indígenas. Revista Electrónica de Investigación Educativa, 20(4), 1-35. Recuperado de https://doi.ora/10.24320/redie.2018.20.4.1169

\section{Resumen}

El artículo explora la construcción identitaria de estudiantes de la Universidad Veracruzana como sujetos que se autoadscriben como indígenas en un contexto histórico específico (post-indigenismo) y las expectativas que despierta esa identificación en el marco de su experiencia formativa a nivel superior. Esta investigación se realizó en el marco de la generación de un diagnóstico participativo que recupera las voces de los estudiantes que se autoadscriben como indígenas sobre su experiencia universitaria y el diseño de un programa institucional intercultural de atención para estudiantes indígenas acorde con sus demandas y necesidades. Institucionalmente se planteó la necesidad de cuestionar la idea de la compensación para la superación de un déficit académico de los estudiantes como premisa de los programas; егa necesario repensar la forma de concebirlos y de actuar en torno a ellos tomando en cuenta sus propias voces y experiencias para el diseño e implementación de un programa no compensatorio.

Palabras clave: Diversidad cultural, política educativa, educación intercultural.

\begin{abstract}
This paper explores identity construction in students from the Universidad Veracruzana as subjects who self-identify as indigenous in a specific historical context - post-indigenism - and the expectations that this generates in the context of their higher education. This study was performed as part of a participatory diagnosis that listens to the voices of students who self-identify as indigenous with respect to their university experience and the design of an institutional, intercultural support service for indigenous students in line with their requests and needs. At an institutional level, a need was expressed to challenge the idea of using compensation as the basis for programs in order to overcome an academic deficit in students. It was necessary to rethink, within the institution, the way we view and act with regard to students, based on their own voices and experiences, in the design and implementation of a noncompensatory program.
\end{abstract}




\section{Introducción}

Este artículo indaga la construcción identitaria de jóvenes estudiantes de la Universidad Veracruzana (UV) como sujetos que se autoadscriben como indígenas en un contexto histórico específico (postindigenismo) y las expectativas que despierta esa identificación en el marco de su experiencia formativa a nivel superior. El punto de partida es la participación en el equipo de trabajo que se propuso plantear un programa pertinente y específico para estudiantes de las regiones urbanas donde se ubican los campus de la uv, atendiendo a una política universitaria que busca favorecer la interculturalización. Visualizando esa necesidad institucional, se planteó: 1) generar un diagnóstico participativo que recuperara las voces de los estudiantes que se autoadscriben como indígenas sobre su experiencia universitaria y 2) diseñar un programa de estudiantes indígenas con enfoque intercultural acorde con sus demandas y necesidades.

En el proceso de trabajo con los jóvenes surgió un tema relevante para ellos: su identificación como indígenas en su condición de estudiantes universitarios en un contexto distinto al de su origen. Este contexto no es sólo institucional sino más amplio, en el cual viven, se forman, se relacionan y, en muchas ocasiones, se establecen permanentemente. Su "migración" por razones académicas es interpretada de múltiples maneras, tanto en sus comunidades como en los contextos a los que llegan: éxito, abandono de sus raíces, prestigio, desapego de su comunidad, formación de élites indígenas, entre muchas otras (Mato, 2008). Las tensiones que se generan dentro y fuera de la universidad influyen en la construcción identitaria de los estudiantes indígenas. Éstas pueden expresar dificultades para insertarse en la institución universitaria y una nueva ciudad, en formas de racismo y discriminación que en ocasiones los conducen a negar su identidad, sus saberes, su lengua. En el marco de estas tensiones se planteó el objetivo inicial de generar de manera colaborativa un programa pertinente para ellos.

Se puso sobre la mesa la necesidad de cuestionar la idea de la compensación para la superación de un déficit académico como premisa de los programas de atención a estudiantes indígenas. Era necesario replantear la forma de concebirlos y de actuar en torno a ellos tomando en cuenta sus propias voces y experiencias como jóvenes indígenas universitarios. En estudios sobre este tipo de programas se habían planteado ya retos tales como la inclusión activa de los estudiantes indígenas en el diseño y operación de los programas, el fortalecimiento de espacios de interacción y la profundización del alcance de este tipo de programas en las instituciones universitarias (Barrón, 2008). Para realizar el proyecto se planteó una metodología basada en la organización de talleres participativos con temáticas específicas para la generación del programa, convocando a estudiantes que se autoadscriben como indígenas.

En primera instancia, el artículo explora la política institucional universitaria de atención a estudiantes indígenas en el marco de tres políticas relacionadas: 1) una política internacional y nacional de acción afirmativa fundada en la premisa de la compensación para el déficit académico de los estudiantes indígenas y con el enfoque intercultural, 2) la política de transversalización de la interculturalidad enunciada en el Programa de Trabajo de la Universidad Veracruzana 2009-2013 y 3) las universidades interculturales. Luego se describe la metodología de trabajo, que incluyó una convocatoria abierta a estudiantes indígenas, la realización de talleres participativos y el análisis de los datos generados. Por último se exponen los hallazgos en relación con los significados atribuidos a ser indígena en la universidad en un proceso continuo de construcción identitaria en medio de un contexto caracterizado por tensiones, así como sus propuestas concretas en torno a lo que la universidad debe considerar para constituirse como un espacio donde la diversidad es reconocida más allá de hacerlo discursivamente en su política institucional.

\subsection{Políticas educativas universitarias}

El difícil acceso y permanencia de los estudiantes indígenas con déficit académico en las universidades públicas constituye un problema social producido a través de categorizaciones que "tienen poderosos efectos de realidad porque estructuran las percepciones, las prácticas y los posibles efectos sociales de los distintos agentes e instituciones" (Martín-Criado, 2007, p. 251). Los efectos en la realidad son de mayor envergadura cuando se generan políticas y dispositivos institucionales para atenderlos. Asimismo, es innegable que la deficiente calidad de la educación recibida por estudiantes indígenas que aspiran a 
ingresar a las universidades, así como las condiciones materiales existentes en las regiones indígenas que habitan, tienen efectos negativos tanto al momento de su acceso como de su permanencia en instituciones de educación superior (Martínez-Casas, 2011). El planteamiento de este problema social a nivel internacional generó una serie de políticas que fueron migrando hasta llegar al contexto local de estudio y, con ellas, se generaron dinámicas, etiquetas, tensiones, necesidad de soluciones a ese problema construido socialmente. Esta exploración de políticas educativas es relevante para explicitar lo que pensamos sobre ellos y cómo actuamos en torno a ellos (Pérez-Ruiz y Valladares, 2014). Este tipo de análisis antropológico de las políticas educativas es fundamental para identificar cómo son definidos los sujetos "destinatarios" de las políticas (Shore y Wright, 1997), dilucidar las implicaciones de esas definiciones en los programas que los atienden y confrontar esas definiciones con los propios sujetos.

Las políticas educativas internacionales y nacionales de acción afirmativa financiadas por Fundación Ford concibieron a los estudiantes indígenas como portadores de un déficit académico que requiere acciones compensatorias. Se planteó que sus trayectorias escolares estaban determinadas por factores de exclusión y rezago que complicaban su acceso a la educación superior. Aunado a lo anterior, les concibió como sujetos individuales para apoyar sus trayectorias escolares y profesionales personales y no como sujetos colectivos, lo cual obstaculiza su organización como grupo en los espacios universitarios. El trasfondo y las implicaciones políticas del programa con relación a la emergencia de una intelectualidad indígena y su posicionamiento en sus regiones, en los movimientos indígenas, en los gobiernos, y en la misma universidad no han sido analizadas en los estudios sobre acción afirmativa (Mateos, Mendoza y Dietz, 2013).

El Programa de Apoyo a Estudiantes Indigenas en Instituciones de Educacion Superior (PAEIIES) fue un programa financiado por la Fundación Ford y coordinado por la Asociación Nacional de Universidades e Instituciones de Educación Superior (ANUIES) que propició la operación de programas de atención de estudiantes indígenas en las universidades públicas teniendo a la tutoría como elemento central para la compensación de su déficit académico. El objetivo fue la permanencia y egreso de los estudiantes indígenas universitarios. Adicionalmente se buscaba la "integración" de los estudiantes a la vida universitaria intentando coadyuvar a su adaptación. Estudios y evaluaciones del programa han rastreado su operación y efectos en los estudiantes atendidos, aunque sin cuestionar de fondo su carácter remedial y compensatorio (Didou y Remedi, 2006, 2009; Flores y Barrón, 2006; Romo, 2006; ANUIES, 2006; González y Velasco, 2012).

La uV operó el programa en la región Xalapa desde 2002 a través de la Unidad de Apoyo Académico a Estudiantes Indígenas (UNAPEI). Badillo (2008), en su estudio sobre los programas de tutorías a estudiantes indígenas en la UNAPEI, apunta a una acepción institucional de lo intercultural que se relacionaba, en ese momento, con un déficit cultural que es necesario compensar para integrarse y funcionar de forma adecuada en el contexto universitario. El enfoque intercultural no era un elemento central ni claro en su abordaje ni implementación. Uno de los dilemas que se han planteado de este tipo de programas ha sido: ¿Nivelador académico o promotor de la interculturalidad? (Flores y Barrón, 2006).

En la uv el significado de lo intercultural se ha ido nutriendo con el tiempo por medio de la reflexión que se generó cuando la UNAPEI se integró a la Universidad Veracruzana Intercultural (UVI). En esa nueva ubicación institucional, lo intercultural se encaminó -como se verá más adelante- a la atención de una gama de diversidades a través de su transversalización bajo la premisa de "interculturalidad para todos". Como enfatiza Ruiz-Lagier (2014): "Las universidades públicas 'convencionales' han introducido discursivamente una noción de interculturalidad que sólo se refiere a una cuestión de intercambios simbólicos y aprecio por las diversas culturas" (p. 90). Sigue quedando pendiente la profundización sobre el vínculo entre interculturalidad y acción afirmativa tanto en las experiencias concretas como a través de análisis prospectivos y propositivos.

En 2005, la uv se convirtió en la primera y única universidad pública en auspiciar en su seno una universidad intercultural en el marco de la política educativa coordinada por la Coordinación General de Educación Intercultural y Bilingüe (CGEIB). La uv adoptó el reto de implementar dos políticas educativas de nivel superior que emergen ante las demandas concretas de los pueblos indígenas como respuestas del Estado mexicano: 1) acción afirmativa para estudiantes indígenas en universidades públicas a través de 
PAEIIES, y 2) universidades interculturales con la oferta de programas considerados pertinentes para y en las regiones indígenas y distintos a los convencionales de las universidades públicas (Dietz y Mateos, 2014).

En 2009, la UVI insertó en su estructura al UNAPEI y la convirtió en UEA con presencia en todas las regiones de la UV. Al interior de las UEA se generaron procesos de reflexión sobre su papel en la universidad y el significado de la transversalización de la interculturalidad con preguntas sobre la pertinencia de: a) atender a la población indígena universitaria a través de la tutoría y/u otras acciones, y b) usar la categoría "indígena" para convocar a estudiantes, ampliar el campo de acción hacia la atención de una diversidad de diversidades a través de la docencia, investigación, etc. Se observó un vuelco a la oferta de los cursos electivos antes mencionados como una herramienta de transversalización de la interculturalidad. Simplificando el panorama, en la uv se han concebido dos tipos de estudiantes indígenas: 1) estudiantes en facultades en programas convencionales con déficit académico que requieren compensación, y 2) estudiantes que requieren de programas académicos sui generis con enfoque intercultural en sus propias regiones. En paralelo surge un tercer tipo, el estudiante no indígena como sujeto a interculturalizarse a través de cursos electivos que lo sensibilicen ante la diversidad. Para cada tipo de estudiante se plantean acciones distintas y, en gran medida, desarticuladas.

\section{Método}

Como parte del equipo de la Unidad de Transversalización Académica Intercultural (UTAI), la tarea como investigadora consistió en detonar un proceso de reflexión con estudiantes indígenas ante la ausencia de un programa específico en operación. Esto llevó a cuestionar si los estudiantes lo consideran necesario y, de ser así, qué características debería tener desde su perspectiva.

Numerosas interrogantes de corte teórico-práctico emergen de la experiencia de trabajo con estudiantes indígenas en el contexto universitario, las cuales llevaron a considerar, previo a este estudio, otros temas pendientes de ser investigados: 1) la definición que las IES hacen del estudiante indígena para su inclusión en programas de corte afirmativo; 2) la indagación cualitativa con estudiantes indígenas sobre su identidad étnica y su uso estratégico en el contexto universitario; 3) la exploración de significados de ser estudiante indígena en una universidad a partir de estudios cualitativos; 4) la revisión de hipótesis tendientes a equiparar estudiantes indígenas y provenientes de zonas rurales, estudiantes reprobadores y estudiantes pobres (Moya, 2013).

Algunos estudios sobre los programas de atención a estudiantes indígenas (Ruiz-Lagier, 2011; Barrón, 2008) coinciden en el diagnóstico de problemáticas comunes a las universidades públicas, tales como la negación de la identidad indígena en el contexto universitario y profesional, discriminación y necesidad de educación intercultural para indígenas y no indígenas. Fue evidente que se requería un proceso de investigación aparejado a este proceso de diseño participativo del programa.

En una primera fase se convocó a estudiantes de la uv hablantes de alguna lengua indígena a diseñar un programa que trascendiera el componente tutorial, y hacerlo en el marco de la UVI aprovechando su experiencia de educación intercultural. A esta convocatoria acudieron 75 estudiantes de la región Xalapa; de los aproximadamente 300 estudiantes invitados a través de correo electrónico, asistieron estudiantes de 15 programas de licenciatura y de 2 posgrados. La invitación fue a participar en cinco talleres con temáticas predefinidas: 1) tutorías para estudiantes indígenas, 2) formación continua (desde la uvı y las UTAI), 3) centro de información y formación de redes, 4) vinculación entre facultades, institutos y UVI, 5) identidad y saberes: reposicionamiento en la uv.

En una segunda fase se celebraron los cinco talleres con una participación voluntaria de 15 estudiantes, en promedio, por taller. Se plantearon dinámicas para propiciar la reflexión colectiva de temas emergentes, la posibilidad de compartir experiencias y la generación de propuestas concretas para el programa. En cada taller se registraron datos a través de relatorías detalladas, notas de campo derivadas de la observación del desarrollo de los talleres y rotafolios en los que cada equipo registraba sus propias reflexiones para compartirlas con el grupo. La sistematización de los datos cualitativos emergentes se 
orientó inicialmente a identificar los insumos para el diseño del programa, pero pronto fue evidente que en la riqueza de los datos emergían como fundamentales dos categorías de análisis: su construcción identitaria y los significados de ser indígena en la universidad. A partir de estas categorías se analizaron los datos partiendo de una estrategia inductiva en la que surgieron subcategorías inspiradas en la teoría fundamentada. El ejercicio de categorización se realizó con el programa ATLAS.ti.

Somos conscientes de que la participación y la consulta pueden constituir prácticas retóricas y simuladoras que legitiman programas diseñados desde arriba, o que no logran articular las demandas, necesidades y expectativas de los que algunos llaman "destinatarios" o "clientes", con las reales posibilidades de las instancias de ejecutarlas con voluntad política, recursos financieros y humanos capacitados y comprometidos.

\section{Discusión}

\subsection{La construcción identitaria de los universitarios indígenas}

La construcción identitaria es un proceso de autocomprensión que retoma los recursos culturales disponibles en un contexto histórico particular (Escobar, 2007). El post-indigenismo caracteriza este momento histórico y político en el que la reivindicación de la diversidad y los derechos de los pueblos indígenas son centrales, al menos discursivamente. Para conceptualizar identidad, Escobar (2007) afirma que el poder y la cultura son negociados en la intersección entre la persona y la sociedad, de forma que se evidencia claramente lo estructural y lo agencial del proceso de autocomprensión. Es particularmente importante analizar la confluencia de lo individual y lo social en el contexto universitario y cómo se articulan las condiciones estructurales y la agencia de los sujetos individuales y colectivos en la construcción de sus identidades y las implicaciones de ello. Esta producción de identidades implica la construcción de mundos localmente situados, culturalmente construidos y socialmente organizados que visibilizan la agencia de los sujetos -tanto individuales como colectivos- y su capacidad de rehacer el mundo en el que habitan y, por supuesto, las instituciones de las que forman parte. Estos mundos relativamente duraderos son espacios donde se generan identidades personales particulares e identidades colectivas.

La universidad es un espacio de construcción identitaria que se caracteriza por transformarse lentamente. Las condiciones históricas tienen un efecto estructurante; sin embargo, los actores lo trascienden a través de la producción de formas culturales que toman a las mismas condiciones históricas como recursos susceptibles de replanteamientos, de transformación. En este sentido, la universidad es susceptible de ser replanteada. Esto ocurre a través de la participación de los actores en conflictos locales explícitos que son generadores de identidad; a su vez, estos conflictos locales explícitos son parte de luchas históricas de largo aliento y mayor alcance. La localización de la demanda de derechos indígenas y reconocimiento de la diversidad a nivel internacional se expresa en una serie de replanteamientos de lo que es ser indígena y ser universitario, así como de la forma en que la universidad gestiona la diversidad en su interior. Las identidades y las luchas son inacabadas y están en proceso, a pesar de existir períodos de estabilización y habitualización. Si bien el planteamiento de la interculturalidad puede considerarse estable y habitualizado, al menos como práctica discursiva, emergerán luchas de largo alcance y conflictos localizados que reten su vigencia y pertinencia.

Los estudiantes que participaron en este proceso cuestionan la visión del indígena como portador de un déficit académico y de esa manera cuestionan la construcción de este problema social. Ellos buscan apoyo académico para lograr un exitoso tránsito por la universidad en las mismas instancias que los demás estudiante; por ejemplo, a través de tutorías a las que todo estudiante de la uv tiene acceso. Lo anterior supone que el planteamiento de un programa de atención con corte compensatorio de un déficit casi inherente de los estudiantes indígenas no es pertinente desde la perspectiva de los estudiantes. Ellos se perciben como sujetos capaces de transitar por la universidad y como una élite de sus comunidades y regiones por haber accedido a la universidad. Resulta evidente que en el imaginario de los estudiantes indígenas subyace la idea y la experiencia de un trato diferenciado por su pertenencia étnica, por ejemplo, en términos de la calidad de la educación que reciben. En este sentido una estudiante señala: 
"Que ser indígena no sea un factor que impida a nosotros y a los maestros darnos una educación de calidad académica competente".

Los estudiantes reflexionan sobre la complejidad de conjugar el reconocimiento de la diversidad y la equidad, no sólo a nivel de políticas educativas (concretamente universitarias), sino a nivel de las percepciones que los demás tienen de los jóvenes indígenas. Una estudiante hace referencia a su ingreso a la universidad diciendo:

Nosotros somos iguales, es más, somos mejores que algunos de la ciudad que no quedaron en la uv. Cuando vine a presentar examen una persona le dijo a mi tía: 'No va a quedar porque es de ranchito y mi nieta sí va a quedar', y resulta que ella no quedó y yo sí. (Estudiante mujer, Derecho, Huasteca).

En un estudio sobre trayectorias y experiencias escolares de estudiantes indígenas, Ortiz (2009) concluye que las trayectorias escolares de los jóvenes indígenas adscritos a la UNAPEI no fueron tan diferentes de las del resto del alumnado de la uv. Su análisis apunta a que la pertenencia a un grupo étnico no marca una diferencia significativa en los modos a través de los cuales estudiantes indígenas y no indígenas enfrentan en su entrada y su permanencia en la universidad. Esta conclusión se plantea a partir de la noción de trayectoria escolar, la cual es rastreada desde una perspectiva cuantitativa y definida con las siguientes variables: índice de aprobación en ordinario, índice de promoción, promedio, desempeño escolar y número de materias que debió haber aprobado en cierto período (Chain y Jácome, 2007); sin embargo, considero que dicha conclusión es susceptible de ser revisada al concebirse otras categorías más allá de las variables mencionadas, y de una indagación de tipo cualitativa y participativa con los propios estudiantes.

Las expectativas propias y ajenas de ingreso y permanencia en la universidad de los jóvenes indígenas inciden en su construcción identitaria como sujetos individuales y colectivos. La decisión de definirse o no como indígena en el contexto universitario es estratégica. La autoadscripción como indígenas no es útil en un contexto monocultural, racista y donde impera la discriminación. Pareciera que no hay condiciones рага ser estudiante y ser indígena ni en la universidad ni en la sociedad en general. Prevalece su identidad como joven universitario, y se puede suponer que al mismo tiempo se debilita la posibilidad del surgimiento de la de un ciudadano universitario indígena que plantea retos concretos a la universidad a partir de las problemáticas que enfrentan sus regiones. Durante la convocatoria a los talleres un joven preguntaba si podíamos ayudarlo a definir si era indígena o no planteando asuntos a nivel teórico, como la relación entre cultura, lengua e identidad, relacionados con la exigibilidad de sus derechos en caso de autoadscribirse como indígena.

Los criterios de la autoadscripción no es sólo un reto institucional, académico y político (Barrón, 2008), sino existencial para los estudiantes. La definición del indígena ha sido construida por el Estado mexicano a través de múltiples canales, como el de la educación pública, teniendo como prototipo al campesino hablante de una lengua indígena portador de vestidos tradicionales, una imagen folklórica, estática y hasta prehispánica. Una gran parte de los estudiantes que participaron en los talleres eligieron como primer elemento de su presentación ser o no hablantes de una lengua indígena. Aquellos que no la hablan se lamentan y explican las razones, pero también reivindican su autoadscripción. La lengua no es el único criterio que ellos consideran válido para identificarse como indígenas. Una estudiante hizo la siguiente reflexión en este sentido:

Provengo de una zona indígena y no sólo los que hablan una lengua deben ser considerados indígenas porque realmente no acaba ahí. Aunque no lo hablo me siento muy identificada con eso y es una lástima que hay jóvenes que por no sentirse excluidos prefieren negar sus orígenes y decir que no lo son, inclusive se sienten avergonzados. Рara mí es un orgullo decir que soy indígena a pesar de que no hablo el idioma. (Estudiante mujer, Medicina, Totonacapan).

La creación -y operación- de instancias para jóvenes indígenas han demostrado ser positivas en términos de generar procesos de apoyo solidario en ambientes donde las prácticas sociales son hostiles hacia la diversidad, como lo enfatizan algunos estudios y el seguimiento a estudiantes universitarios indígenas 
(Chávez, 2008). Sin embargo, el desdibujamiento de la identidad indígena en este contexto obstaculiza la creación e identificación de redes de apoyo entre estudiantes que provienen de las mismas regiones y comunidades. La negación de su identidad no sólo es funcional para impedir que se configuren como sujetos colectivos en los ámbitos donde interactúan, sino también para el mantenimiento del status quo monocultural y discriminatorio, donde, por ejemplo, las lenguas indígenas no tienen cabida. Los talleres fueron espacios abiertos para compartir experiencias dolorosas que han forjado su tránsito por la universidad: vergüenza por hablar una lengua indígena, discriminación por su origen indígena, pocas expectativas de sus capacidades por parte de los académicos, intento de convertirse en personas distintas a partir de lo socialmente aceptable, entre muchas otras. Sin embargo, los talleres posibilitaron también la reflexión en cuanto a la potencial utilidad de constituirse como un grupo para lograr efectos positivos en su tránsito por la universidad y, en general, en la institución.

Muchos participantes manifestaron que las pocas expectativas en su capacidad no sólo provienen de los no indígenas, sino de ellos mismos. Un estudiante comentó:

Hay veces que nosotros pensamos que por ser parte de una comunidad no podemos llegar hasta acá y yo creo que sí podemos. (Estudiante hombre, Contaduría, Huasteca).

Se identificaron áreas de acción que implican el reconocimiento del valor de sus propias prácticas culturales y de sus lenguas como elementos vivos. Una reflexión es la siguiente:

Venimos a copiar otras cosas que no es nuestra identidad, somos indígenas por naturaleza y tenemos que apropiarnos de eso y hacer un cambio, porque no podemos hablar otra lengua si no hablamos la de nosotros, es como sentirnos apenados por nosotros. (Estudiante hombre, Administración, Zongolica).

En su experiencia formativa, ni la currícula ni los programas de vinculación han sido ámbitos donde las culturas y las lenguas encuentren expresión concreta ni espacios que las potencien. El potencial de la incorporación de los saberes de sus pueblos en la universidad y en su formación es puesto de manifiesto por los estudiantes; desde su perspectiva no es un problema únicamente disciplinario, sino también del tipo de relación de la universidad con los diferentes sectores sociales. Esto sin duda incide en la formación ciudadana de los estudiantes, en la formación de sujetos colectivos, en la relación que construyen con sus comunidades y pueblos al egresar de la universidad, en la formación de élites indígenas funcionales al status quo, entre otros. Estudios que han generado pistas para el replanteamiento de los programas para estudiantes indígenas ponen sobre la mesa la necesidad de que incluyan acciones que "re-arraiguen" y "re-vinculen" a los estudiantes con sus comunidades de origen y sus problemáticas locales y regionales (Medrano y Flores, 2007).

\subsection{Las expectativas de los universitarios indígenas}

Al plantear un diseño participativo para elaborar un programa para estudiantes indígenas emergieron múltiples expectativas. La ciudadanización de los estudiantes no es un proceso automático en la trayectoria universitaria; se requieren estrategias concretas para vivenciar los significados e implicaciones de ser ciudadano. Sin ser la ciudadanización un objetivo principal de la convocatoria al diseño de un programa con ellos y para ellos, su interés en participar generó un proceso que coadyuva a la participación sustantiva en la definición de agendas, la toma de decisiones, la generación de acuerdos y la incidencia política en varios niveles. La demanda y el interés de los estudiantes por participar en este tipo de procesos no son nuevos. Desde la implementación de los programas de atención a estudiantes indígenas con el financiamiento de PAEIIES, una demanda reiterada fue la participación estudiantil para su diseño, implementación y evaluación. Esto pareciera contradecir a estudios que muestran los bajos niveles de participación de los jóvenes en temas emergentes. Es observable que la participación creciente en torno a la problemática indígena por parte de los jóvenes de los propios pueblos; esto se conjuga con el hecho de que las universidades constituyen los espacios donde estos asuntos han cobrado mayor relevancia (Hopenhayn, 2007). 
Un aspecto central que emergió de las reflexiones de los estudiantes fue la intención de conformarse como un grupo de estudiantes indígenas portadores de su identidad en un contexto universitario susceptible de ser transformado a nivel de sus prácticas, materializando su propia política institucional de interculturalización. La formación de sujetos con identidades colectivas plantea un reto importante a las universidades. Del mismo modo que la generación del conocimiento es una actividad eminentemente política que dista de la neutralidad, la formación universitaria está entrelazada a la formación de ciudadanos posicionados políticamente para la transformación de un status quo que puede resultar lacerante para un amplio espectro de la sociedad. Cuando los estudiantes se dieron cuenta de la inexistencia de redes de estudiantes por regiones, comunidades, grupos indígenas, etc., su propuesta fue organizarse formalmente para constituir no sólo instancias autogestivas de apoyo solidario, sino también órganos de vinculación con sus regiones, colectivos con la capacidad de actuar en problemáticas específicas. De modo que la formación de redes y de un grupo organizado como estudiantes indígenas es un acto político que explicita su posicionamiento como ciudadanos. La apertura de espacios de reflexión entre universitarios y universidades es imprescindible para dar respuesta a retos que no son coyunturales, sino estructurales.

Las propuestas de los estudiantes trastocan ejes centrales de las universidades: la formación, la vinculación y la investigación. En cuanto a formación, proponen que los programas académicos sean pertinentes y respondan a las necesidades de sus regiones a través de reformas curriculares. El diseño de nuevos planes de estudio presenta retos relacionados también a la investigación para la incorporación articulada de saberes no científicos ni occidentales, sino tradicionales, generados y transmitidos en contextos no académicos. Un diálogo de saberes al que la universidad debe abrirse y replantear su hegemonía en la generación de conocimiento válido. Sin duda, la emergencia de este eje es novedoso en el ámbito universitario convencional -no el propiamente intercultural- y el más desafiante para la institución educativa desde el punto de vista de los estudiantes.

Asimismo, plantean una nueva perspectiva sobre la vinculación, a la que visualizan en dos direcciones: desde la universidad hacia sus regiones redefiniendo la histórica verticalidad de su vinculación, y desde sus regiones a la universidad para compartir conocimientos locales detonando un proceso dialógico y horizontal. La universidad debe ir a las regiones con una renovada postura sobre lo que es vinculación, para ello requiere escuchar y no imponer relaciones verticales y asistencialistas a las comunidades. La pertinencia social de la vinculación depende también del involucramiento de estudiantes que provienen de esas regiones, de la capacidad de la universidad de reconocer el valor de los diagnósticos, de las problematizaciones y de las soluciones generadas por los propios pueblos. Un estudiante del área de Salud hace hincapié en esta necesidad de espacios de formación que consideren el reconocimiento de la diversidad y las implicaciones para sus propias profesiones, no sólo a nivel teórico, sino heurístico y axiológico:

En el área de la Salud ya se está perdiendo esa culturización y esa humanización del profesional de la salud y entonces cuando llegamos a la práctica con personas en zonas rurales, independientemente de Oaxaca y de cualquier lado, pues es muy difícil prestarle un servicio a esas personas. (Estudiante mujer, Medicina, Tehuantepec).

Es notorio que desde la enunciación de una política de interculturalización de la universidad coordinada por la UTAI de la UVI falta mucho por hacer para lograr una efectiva articulación de objetivos y acciones de facultades y las sedes de la UVI. Esto posibilitaría su articulación para propiciar la movilidad estudiantil y académica, acciones de vinculación pertinente en las regiones, diseño de programas con enfoque intercultural en las facultades, fortalecimiento de programas académicos a través de trabajo académico colaborativo, etc. Los estudiantes expresan la necesidad de conocer la propuesta del enfoque intercultural ante las potencialidades que parecen asomarse con su aplicación. El replanteamiento de la política de vinculación de las universidades implica una revisión de la noción tradicional de relación universidad-sociedad, de los actores universitarios que hacen vinculación, cómo la hacen y para qué, así como de las problemáticas que requieren la atención de los universitarios. 


\section{Conclusiones}

La construcción identitaria de los estudiantes indígenas universitarios está mediada por condiciones históricas y políticas concretas que si bien han generado un contexto adverso, caracterizado por el racismo, la exclusión y la desigualdad, también posibilitan la transformación de las instituciones de educación superior hacia prácticas de gestión de su diversidad. Las políticas institucionales, permeadas por políticas educativas nacionales e internacionales, requieren replantear la definición de estudiante indígena retando su premisa de compensación al déficit casi inherente de estos jóvenes. Escuchar las voces de los estudiantes puede generar pistas claras de la ruta para transitar hacia una universidad que no sea racista ni monocultural. El planteamiento de los estudiantes no se limita a propuestas de corte axiológico que en ocasiones caracteriza el discurso intercultural, sino de una transformación profunda que dé cabida, con equidad, a las múltiples epistemologías, saberes, metodologías, que han sido excluidas de las instituciones de educación superior. Sin duda, esto requiere un trabajo colectivo y colaborativo dentro las instituciones entre sujetos que históricamente han estado fuera de la universidad y sujetos que han ejercido su hegemonía política e intelectual.

Los jóvenes indígenas han sido capaces de complejizar y replantear un problema social construido desde instancias de poder nacional e internacional. Este replanteamiento tiene implicaciones profundas en la forma de definir a la población indígena universitaria y la noción de universidad como espacio multicultural, no sólo por la composición de su comunidad, sino por su potencial capacidad de entablar un diálogo de saberes.

\section{Referencias}

Asociación Nacional de Universidades e Instituciones de Educación Superior (Ed.) (2006). Experiencias de atención a estudiantes indígenas en instituciones de educación superior. México: Autor.

Badillo, J. (2008). La operación de los programas de tutorías en la Universidad Veracruzana y sus efectos en la experiencia escolar. Tesis de maestría. Xalapa: Universidad Veracruzana.

Barrón, J. C. (2008). ¿Promoviendo relaciones interculturales? Racismo y acción afirmativa en México para indígenas en Educación Superior. Trace, 53, 22-35. Recuperado de

http://journals.openedition.org/trace/352

Chain, R. y Jácome, N. (2007). Perfil de ingreso y trayectoria escolar en la Universidad. Veracruz: Universidad Veracruzana.

Chávez, M. E. (2008). Ser indígena en la educación superior. ¿Desventajas reales o asignadas? Revista de la Educación Superior, 4(148), 31-55.

Didou, S. y Remedi, E. (2006). Pathways to higher education: una oportunidad de educación superior para jóvenes indígenas en México. México: ANUIES, CINVESTAV.

Didou, S. y Remedi, E. (2009). Los olvidados: acción afirmativa de base étnica e instituciones de educación superior en América Latina. México: Juan Pablos Editores, CINVESTAV.

Dietz, G. y Mateos, L. S. (2014). La Universidad Veracruzana Intercultural (UvI): una mirada desde su acompañamiento etnográfico. En S. Martí y G. Dietz (Eds.). Empoderamiento y educación superior en contextos interculturales en México (pp. 79-94). Barcelona: Bellaterra.

Escobar, A. (2007). Identity. En D. Nugent y J. Vincent (Eds.). A companion to the anthropology of politics (pp. 248-266). Oxford: Blackwell Publishing. 
Flores, P. y Barrón, J. C. (2006). El programa de apoyo a estudiantes indígenas: ¿nivelador académico o impulsor de la interculturalidad? México: ANUIES.

González, A. P. y Velasco, S. (Coords.) (2012). Prácticas tutoriales con estudiantes indígenas. Experiencias y propuestas de trabajo en instituciones mexicanas de educación superior. México: ANUIES.

Hopenhayn, M. (2007). Participación juvenil. En M. Zuleta, H. Cubides y M. R. Escobar (Comps.). ¿Uno solo o varios mundos?: diferencia, subjetividad y conocimientos en las ciencias sociales contemporáneas (pp. 231246). Bogotá: Siglo del Hombre.

Mateos, L. S., Mendoza, R. G. y Dietz, G. (2013). Diversidad e interculturalidad en la educación superior convencional. En M. Bertely, G. Dietz y M. G. Díaz (Coords.). Multiculturalismo y educación 2002-2011 (pp. 307-348). México: ANUIES, COMIE.

Martín-Criado, E. (2007). La juventud como apuesta política. En M. Zuleta, H. Cubides y M. R. Escobar (Comps.). ¿Uno solo o varios mundos?: diferencia, subjetividad y conocimientos en las ciencias sociales contemporáneas (pp. 247-262). Bogotá: Siglo del Hombre.

Martínez-Casas, R. (2011) La formación de los profesionistas bilingües indígenas en el México contemporáneo. Perfiles Educativos, XXXIII, 250-261.

Mato, D. (2008). Diversidad cultural e interculturalidad en educación superior. Problemas, retos, oportunidades y experiencias en América Latina. En D. Mato (Ed.). Diversidad Cultural e Intercultural en Educación Superior. Experiencias en América Latina (pp. 21-82). Venezuela: unEsco.

Medrano, T. F. y Flores, T. (2007). Programa de apoyo a estudiantes indígenas. Sistematización de la experiencia de tutorías. Guadalajara: UDG-UACI.

Moya, R. (2013). El acceso y la inclusión de indígenas y afrodescendientes en las universidades latinoamericanas. Pueblos Indígenas y Educación, 63, 31-104.

Ortiz, V. (2009). Las trayectorias y experiencias escolares de los estudiantes de origen indigena de la Universidad Veracruzana. Tesis de maestría, Universidad Veracruzana, Veracruz, México.

Pérez, M. L. y Valladares, L. R. (2014). Introducción. Historicidad y actualidad de las juventudes indígenas en América Latina. En M. L. Pérez y L. R. Valladares (Coords.). Juventudes indígenas: De hip hop y protesta social en América Latina (pp. 9-34). México: INAH.

Romo, A. (2006). Evaluación del programa de tutoría a estudiantes indígenas. México: ANUIES.

Ruiz-Lagier, V. (2011). Las mujeres indígenas y su acceso a la educación superior, dos estudios de caso. En S. Didou y E. Remedi (Coords.). Educación Superior de carácter étnico en México: pendientes para la reflexión (pp. 103-126). México: Senado de la República, CINVESTAV.

Ruiz-Lagier, V. (2014). ¿Cómo introducir el debate intercultural en los espacios 'convencionales' de educación superior? En M. L. Pérez, V. Ruiz-Lagier y S. Velasco (Coords.). Interculturalidad(es). Jóvenes indigenas y migración (pp. 77-106). México: UPN.

Shore, C. y Wright, S. (1997). Policy: a new field of anthropology. En C. Shore y S. Wright (Eds.). Anthropology of policy. Critical perspectives on governance and power (pp. 3-42). Nueva York: Routledge.

Universidad Veracruzana Intercultural. (2013). Unidad de transversalización académica intercultural. Manuscrito no publicado. Xalapa: Autor. 